\title{
Towards Concise Representation for Taxonomies of Epistemic Communities
}

\author{
Camille Roth*, Sergei Obiedkov ${ }^{\dagger}$, and Derrick G. Kourie ${ }^{\dagger}$
}

\begin{abstract}
We present an application of formal concept analysis aimed at creating and representing a meaningful structure of knowledge communities under the form of a lattice-based taxonomy built upon groups of agents jointly manipulating some notions. The resulting structure is however usually extremely complex, hence uneasy to comprehend. We consider two approaches to build a concise representation respecting the original taxonomy, while hiding uninteresting and superfluous information, using both a pruning strategy based on the notion of concept stability and a representational improvement based on nested line diagrams. We illustrate the method with a small community of embryologists.
\end{abstract}

\section{Introduction}

A knowledge community is a group of agents who produce and exchange knowledge within a given knowledge field, achieving a widespread social cognition task in a rather decentralized, collectively interactive, and networked fashion. The study of such communities is usually a focal topic for social epistemology as well as scientometrics and political science $[1,2,3]$, inter alia.

In particular, a traditional concern relates to the description of the structure of knowledge communities [4] generally organized in several subcommunities. In contrast to the limited, subjective, and implicit representation that agents have of their own global community - a folk taxonomy [5] - epistemologists typically use expertmade taxonomies, more reliable but still falling short of precision, objectivity, and comprehensiveness.

We describe here an application of formal concept analysis (FCA) aimed at representing a meaningful structure of a given knowledge community under the form of a lattice-based taxonomy built upon groups of agents jointly manipulating some notions. Formal concepts in this case relate loosely to the sociological notion of "structural equivalence" [6], denoting groups of agents linked jointly to a certain set of terms.

This work is a development of the approach from $[7,8]$, which is briefly described in Sect. 2. In Sect. 3, we concentrate on how to make lattice-based taxonomies concise and intelligible. Concept lattices faithfully represent every trait in data, including those due to noise. Therefore, we need tools that would allow us to abstract from insignificant and noisy traits. To this end, we suggest a pruning technique based on stability indices of concepts [9] and apply it on its own and in combination with nested line diagrams [10]. The latter allows representing the community structure at various levels of precision. The techniques described in Sect. 3 admit modifications, which are a subject for further research and experiment. Some possible directions are listed in Sect. 4.

\section{A Formal Concept Analysis Ap- proach in Applied Epistemology}

\section{$2.1 \quad$ Framework}

Representing taxonomies of knowledge communities has usually been an issue for applied epistemology and scientometrics [2], addressed notably by describing community partitions with trees or two-dimensional maps of agents and topics. Various quantitative methods are being used, often based on categorization techniques and data denoting links between authors, papers and/or notions - such as co-citation [4], co-authorship [11], or co-occurrence data [12].

In contrast, lattice-based taxonomies we discuss here allow overlapping category building, with agents possibly belonging to several fields at once, and render a finer structure of knowledge fields by representing various kinds of interrelationships. Our notion of community is both looser and more general than the sociological notion of structural equivalence [6]: we identify groups of agents linked jointly to various sets of notions instead of exactly the same notions.

\footnotetext{
*Department of Social and Cognitive Science, University of Modena and Reggio Emilia, Italy, and Center of Research in Applied Epistemology, CNRS/Ecole Polytechnique,Paris, France, camille.roth@polytechnique.edu

${ }^{\dagger}$ Department of Computer Science, University of Pretoria, Pretoria, South Africa, sergei.obj@gmail.com and dkourie@cs.up.ac.za
} 
A similar problem of identifying communities exists in the area of social networks. Lattices have also been used there $[13,14,15]$, but in that context, groups of actors are generally considered to be disjoint and a lattice is only a first step in their construction. Besides, social aspects of the community structure-leaders, peripheral members, cooperation within and between different groups, etc.are usually of prime interest, whereas we rather try to discover a structure of a scientific field without focusing on individuals. Because of these different accents, social network lattices are typically based on data describing interactions between actors, while our data describes actors in terms of the domain for which we want to build a taxonomy.

Before proceeding, we briefly recall the FCA terminology [10]. Given a (formal) context $\mathbb{K}=(G, M, I)$, where $G$ is called a set of objects, $M$ is called a set of attributes, and the binary relation $I \subseteq G \times M$ specifies which objects have which attribute, the derivation operators $(\cdot)^{I}$ are defined for $A \subseteq G$ and $B \subseteq M$ :

$$
\begin{aligned}
& A^{I}=\{m \in M \mid \forall g \in A: g \operatorname{Im}\} ; \\
& B^{I}=\{g \in G \mid \forall m \in B: g \operatorname{Im}\} .
\end{aligned}
$$

In words, $A^{I}$ is the set of attributes common to all objects of $A$ and $B^{I}$ is the set of objects sharing all attributes of $B$.

If this does not result in ambiguity, $(\cdot)^{\prime}$ is used instead of $(\cdot)^{I}$. The double application of $(\cdot)^{\prime}$ is a closure operator, i.e., $(\cdot)^{\prime \prime}$ is extensive, idempotent, and monotonous. Therefore, sets $A^{\prime \prime}$ and $B^{\prime \prime}$ are said to be closed.

A (formal) concept of the context $(G, M, I)$ is a pair $(A, B)$, where $A \subseteq G, B \subseteq M, A=B^{\prime}$, and $B=A^{\prime}$. In this case, we also have $A=A^{\prime \prime}$ and $B=B^{\prime \prime}$. The set $A$ is called the extent and $B$ is called the intent of the concept $(A, B)$.

A concept $(A, B)$ is a subconcept of $(C, D)$ if $A \subseteq C$ (equivalently, $D \subseteq B$ ), then $(C, D)$ is called a superconcept of $(A, B)$. We write $(A, B) \leq(C, D)$ and define the relations $\geq,<$, and $>$ as usual. If $(A, B)<(C, D)$ and there is no $(E, F)$ such that $(A, B)<(E, F)<(C, D)$, then $(A, B)$ is a lower neighbor of $(C, D)$ and $(C, D)$ is an upper neighbor of $(A, B)$; notation: $(A, B) \prec(C, D)$ and $(C, D) \succ(A, B)$.

The set of all concepts ordered by $\leq$ forms a lattice, which is denoted by $\underline{\mathfrak{B}}(\mathbb{K})$ and called the concept lattice of the context $\mathbb{K}$. The relation $\prec$ defines edges in the covering graph of $\underline{\mathfrak{B}}(\mathbb{K})$.

\subsubsection{Epistemic Community Taxonomy.}

Our primary data consists of scientific papers dealing with a certain (relatively broad) topic, from which we construct a set $G$ of authors and a set $M$ of notions used in these papers. Thus, we have a context $(G, M, I)$, where $I$ describes which author uses which term in one of his or her papers: $g I m$ iff $g$ uses $m$. Then, for a group of authors $A \subseteq G, A^{\prime}$ represents notions being used by every author $a \in A$, while, for a set of notions $B \subseteq M, B^{\prime}$ is the set of authors using every notion $b \in B$. We see notions as cognitive properties of authors who use them (skills in scientific fields) and authors as extents of notions.

The intent of a concept in this context is a subtopic and the extent is the set of all authors active in this subtopic. Thus, formal concepts provide a solid formalization of the notion of epistemic community (EC), traditionally defined as a group of agents dealing with a common set of issues and a common goal of knowledge creation [3]. By EC, we understand henceforth a field or a subdiscipline together with its authors, irrespective of their affiliation or personal interactions, i.e., neither a department nor a research project. The concept lattice represents the structure of a given knowledge community as a taxonomy of ECs, with more populated and less specific subtopics closer to the top [7].

\subsection{Empirical Example and Protocol}

We focus on a bibliographical database of MedLine abstracts coming from the fast-growing community of embryologists working on the zebrafish during the period 1998-2003. ${ }^{1}$ We build up a context describing which author used which notion during the whole period, where the notion set is made of a limited dictionary of about 70 lemmatized words selected by the expert [16] among the most frequent yet significant words of the community, i.e., excluding rhetorical and paradigmatic words such as "is", "with", "study", "biology", "develop", etc. Then, we extract a random sample context of 25 agents and 18 words, which we use to illustrate the techniques described in the paper. The concept lattice of this context is shown in Fig. 1 (only attribute labels are shown); it contains 69 formal concepts or epistemic communities ${ }^{2}$.

We use an expert-based description of the zebrafish community taxonomy as a benchmark for our procedure $[16,17,18]$. Three major subfields are to be distinguished. First, an important part of the community focuses on biochemical signaling mechanisms, involving pathways and receptors, which are crucial in understanding embryo growth processes. A second field includes comparative studies: the zebrafish, as a model animal, may show similarities with other close vertebrate species, in particular, mice and humans. Finally, another significant area of interest relates to the brain and the nervous system, notably in association with signaling in brain development.

\footnotetext{
${ }^{1}$ Data is obtained from a query on article abstracts containing the term "zebrafish" at http://www.pubmed.com. Using a precise term is likely to delimit properly the community, in contrast to global terms such as "molecular biology".

${ }^{2}$ Diagrams are produced with ConExp (http://sourceforge.net/projects/conexp) and ToscanaJ (http://sourceforge.net/projects/toscanaj).
} 


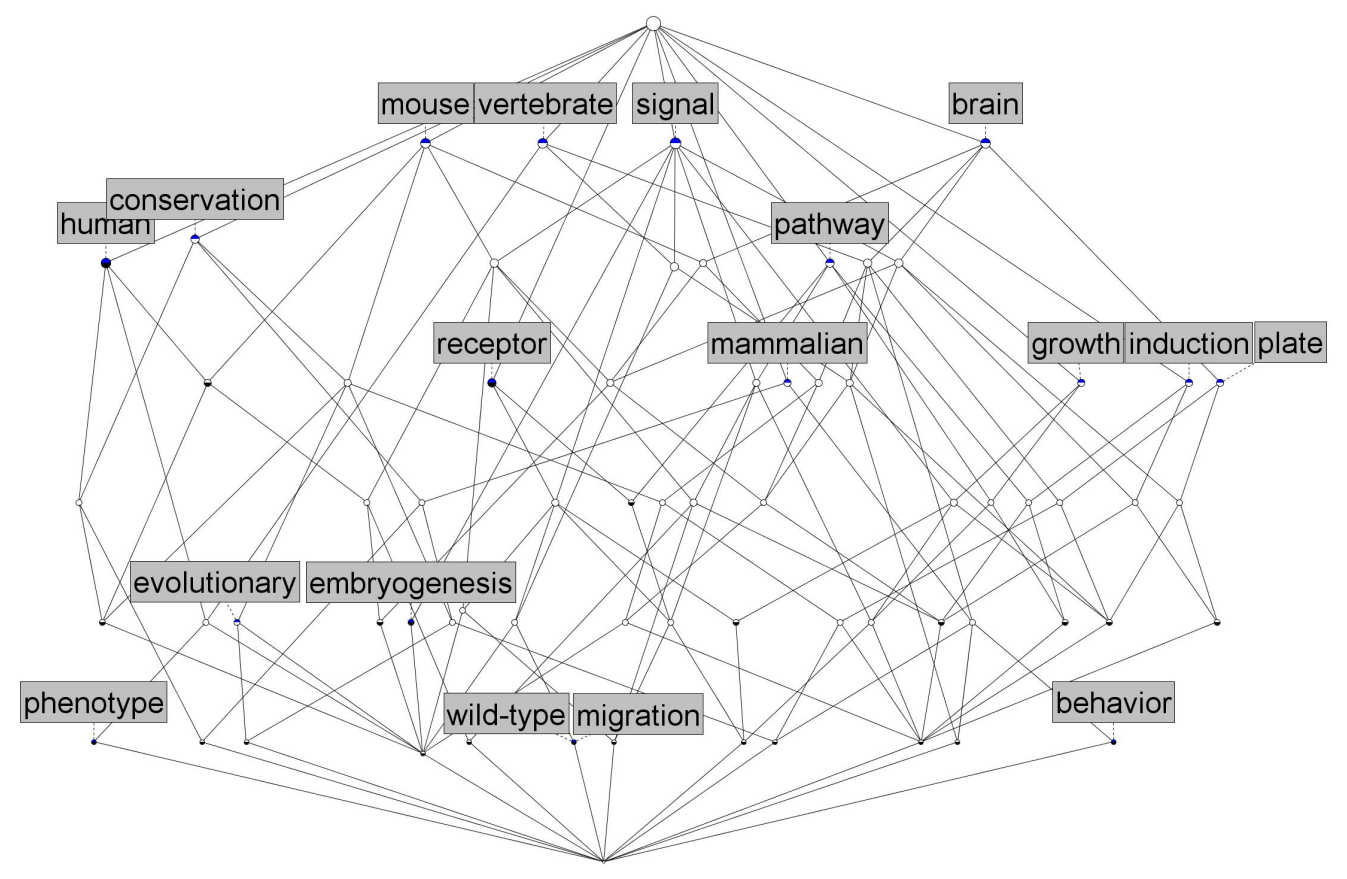

Figure 1: The concept lattice of a sample zebrafish context.

\section{Concise Representation}

\subsection{Rationale}

Even if the concept lattice appears to adequately organize these communities, the number of ECs is still likely to be large. Although derived from a small context, the diagram in Fig. 1 is indeed rather complicated. This caveat affects concept lattices in general, which structure the data but do not reduce it much. To quote [10], "even carefully constructed line diagrams lose their readability from a certain size up".

In this respect, some ECs are likely to be irrelevant to describe the taxonomy of knowledge fields. One solution is to compute only an upper part of the lattice (an order filter), e.g., concepts covering at least $n \%$ of authors (in this case, we get an "iceberg lattice" [19]). However, to take into account small but interesting groups as well, typical for example of heteredox topics or new yet still minor trends, one should also compute all lower neighbors of "large" ECs (satisfying the $n \%$ threshold). Top-down lattice construction algorithms are particularly suitable for this approach [20]; alternatively, one may look at algorithms designed specifically for constructing iceberg lattices [19] and other algorithms from the frequent itemset mining community [21]. The reduction in the number of concepts can be considerable; however, though computationally feasible, this would still be unsatisfying from the standpoint of manual analysis.

Clearly, the size of the concept lattice is not only a computational problem. The lattice may contain nodes that are just too similar to each other because of noise in data or real minor differences yet irrelevant to our purposes. In this case, taking an upper part of the lattice does not solve the problem, since this part may well contain such similar nodes. Besides, it should also be interesting to distinguish major trends from minor subfields; that is, having a representation allowing for different levels of precision.

In this section, we consider two approaches to improve the readability of line diagrams: pruning and nesting. When pruning, we assume that some concepts are irrelevant: we filter out those which do not satisfy specified constraints of a certain kind. In a previous attempt to use concept lattices to represent EC taxonomies [7, 8], heuristics combining various criteria - such as extent size, shortest distance from top, number of lower neighbors, etc. - were used to score ECs and keep only the $n$ best ones. The resulting pictures were meaningful taxonomies but required a posteriori manual analysis, while it was unclear whether it could be possible to go further than a rough rough representation. Here, we focus on a particular pruning strategy based on the notion of stability of a concept [9].

Nested line diagrams [10], on the other hand, provide no reduction and, hence, do not incur any loss of information. Rather, they rearrange the concepts in such a way that the entire structure becomes more readable; they provide the user with a partial view, which can then be extended to a full view if so desired. Thus, nested line diagrams offer a useful technique for representing complex 
structures. Yet, because they preserve all details of the lattice, in order to get rid of (many) irrelevant details we combine nesting and pruning in Sect. 3.4. We thus try to get a representation that respects the original taxonomy while hiding at the same time uninteresting and superfluous information; our aim is a compromise between the noise level, the number of detail, and readability.

\subsection{Stability-Based Pruning}

Our structures are complex, but, in fact, they are more complex than they should be, because our data is fairly noisy: for instance, an author might use a term accidentally (e.g., discussing related work), or different names denote the same thing (e.g., "Galois lattice" and "concept lattice"). As a result, many concepts do not correspond to real communities, and some pruning seems unavoidable.

The pruning technique we describe here is based on the notion of stability first introduced in [22] in relation to hypotheses generated from positive and negative examples; it can be easily extended to formal concepts of a context [9]. The definition we use is slightly different from the original one, but the difference is irrelevant to our discussion.

Definition 1. Let $\mathbb{K}=(G, M, I)$ be a formal context and $(A, B)$ be a formal concept of $\mathbb{K}$. The stability index, $\sigma$, of $(A, B)$ is defined as follows:

$$
\sigma(A, B)=\frac{\left|\left\{C \subseteq A \mid C^{\prime}=B\right\}\right|}{2^{|A|}} .
$$

The stability index of a concept indicates how much the concept intent depends on particular objects of the extent. A stable intent is probably "real" even if the description of some objects is "noisy". In application to our data, the stability index shows how likely we are to still observe a field if we ignore several authors. Apart from noise-resistance, a stable field does not collapse (e.g., merge with a different field, split into several independent subfields) when a few members stop being active or switch to another topic. The following proposition describing the stability index of a concept $(A, B)$ as a ratio between the number of subcontexts of $\mathbb{K}$ where $B$ is an intent and the total number of subcontexts of $\mathbb{K}$ makes more explicit the idea behind stability:

Proposition 1. Let $\mathbb{K}=(G, M, I)$ be a formal context and $(A, B)$ be a formal concept of $\mathbb{K}$. For a set $H \subseteq G$, let $I_{H}=I \cap(H \times M)$ and $\mathbb{K}_{H}=\left(H, M, I_{H}\right)$. Then,

$$
\sigma(A, B)=\frac{\mid\left\{\mathbb{K}_{H} \mid H \subseteq G \text { and } B=B^{I_{H} I_{H}}\right\} \mid}{2^{|G|}} .
$$

Proof. Every $C \subseteq A$ defines a family of contexts:

$$
\mathfrak{F}_{C}(\mathbb{K})=\left\{\mathbb{K}_{H} \mid C \subseteq H \subseteq G \text { and } A \cap H=C\right\} .
$$

Obviously, $\mathfrak{F}_{C}(\mathbb{K}) \cap \mathfrak{F}_{D}(\mathbb{K})=\varnothing$ if $C \neq D$. In fact, the sets $\mathfrak{F}_{C}(\mathbb{K})$ form a partition of subcontexts of $\mathbb{K}$ (with the same attribute $\operatorname{set} M)$. It is easy to see that all sets $\mathfrak{F}_{C}(\mathbb{K})$ (with $C \subseteq A$ ) have the same size: $\left|\mathfrak{F}_{C}(\mathbb{K})\right|=2^{|G|-|A|}$. Note also that, for $\mathbb{K}_{H} \in \mathfrak{F}_{C}(\mathbb{K})$, we have $B^{I_{H} I_{H}}=C^{I_{H}}=$ $C^{\prime}$; hence, $B$ is closed in the context $\mathbb{K}_{H} \in \mathfrak{F}_{C}(\mathbb{K})$ if and only if $C^{\prime}=B$. Therefore,

$$
\left|\left\{\mathbb{K}_{H} \mid H \subseteq G, B=B^{I_{H} I_{H}}\right\}\right|=\frac{2^{|G|}\left|\left\{C \subseteq A \mid C^{\prime}=B\right\}\right|}{2^{|A|}},
$$

which proves the proposition.

In other words, the stability of a concept is the probability of preserving its intent after leaving out an arbitrary number of objects. This is the idea of cross-validation [23] carried to its extreme: stable intents are those generated by a large number of subsets of our data.

Computing Stability. In [9], it is shown that, given a formal context and its concept, the problem of computing the stability index of the concept is \#P-complete. In Tab. 1, we present a simple algorithm that takes the covering graph of a concept lattice $\mathfrak{B}(\mathbb{K})$ and computes the stability indices for every concept of the lattice. The algorithm is meant only as an illustration of a general strategy for computing the stability; therefore, we leave out any possible optimizations.

To determine the stability index $\sigma(A, B)$, we compute the number of subsets $E \subseteq A$ that generate the intersection $B$ (i.e., for which $E^{\prime}=B$ ) and store it in Subsets. $\sigma(A, B)$ is simply the number of such subsets divided by the number of all subsets of $A$, that is, by $2^{|A|}$. Once computed, $\sigma(A, B)$ is stored in Stability, which is the output of the algorithm.

The algorithm traverses the covering graph from the bottom concept upwards. A concept is processed only after the stability indices of all its subconcepts have been computed; the Count variable is used to keep track of concepts that become eligible for processing. In the beginning of the algorithm, Count $[(A, B)]$ is initialized to the number of lower neighbors of $(A, B)$. When the stability index is computed for some lower neighbor of $(A, B)$, we decrement Count $[(A, B)]$. By the time Count $[(A$, B)] reaches zero, we have computed the stability indices for all lower neighbors of $(A, B)$ and, consequently, for all subconcepts of $(A, B)$. Then, it is possible to determine the stability index of $(A, B)$.

Initially, Subsets $[(A, B)]$ is set to the number of all subsets of $A$, that is, $2^{|A|}$. Before processing $(A, B)$ we process all subconcepts $(C, D)$ of $(A, B)$ and decrement Subsets [(A, B) ] by the number of subsets of $C$ generating the intersection $D$. By doing so, we actually subtract from $2^{|A|}$ the number of subsets of $A$ which do not generate $B$ : indeed, every subset of $A$ generates either $B$ or the intent of a subconcept of $(A, B)$. Thus, the value of Subsets $[(A, B)]$ eventually becomes equal to the number of subsets of $A$ generating $B$. 


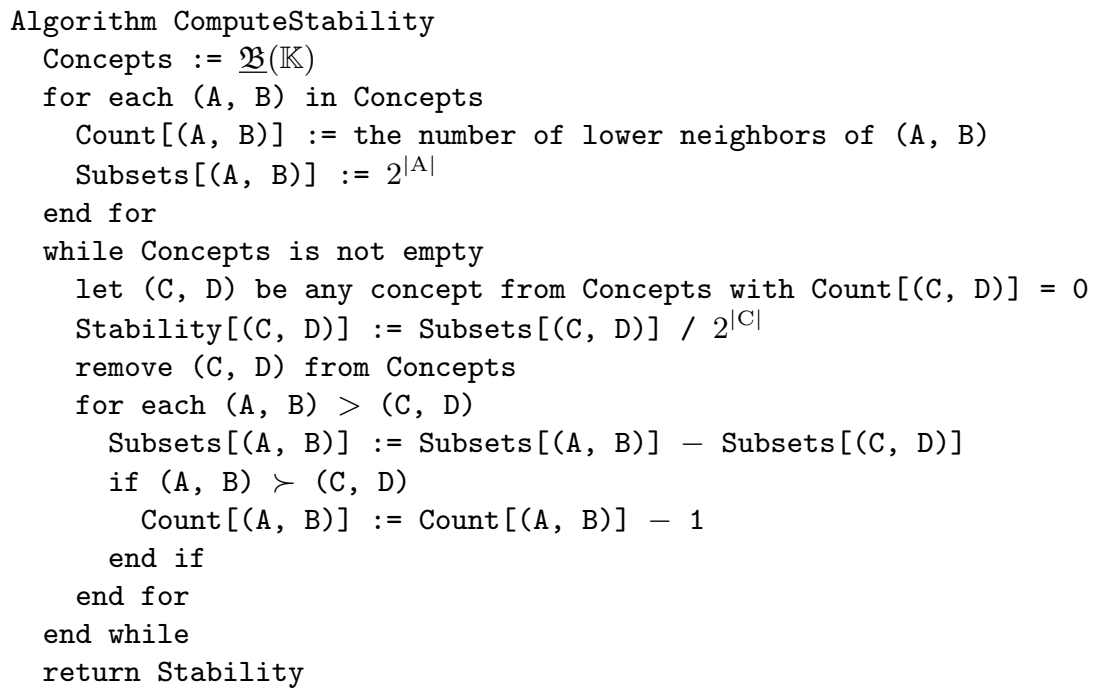

Table 1: Algorithm to compute stability.

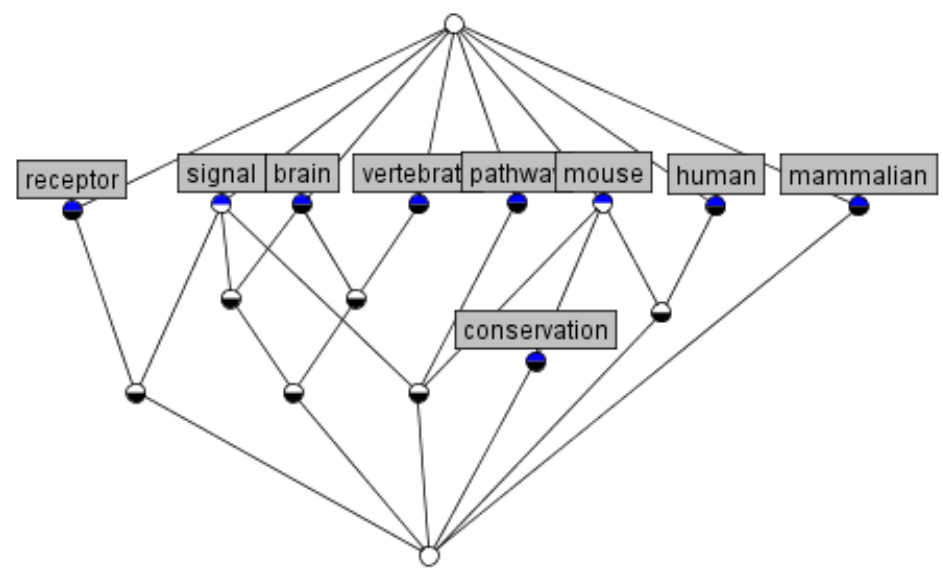

Figure 2: The pruned lattice of Fig. 1, with stability threshold 0.52.

Applying Stability. The basic stability-based pruning method is to remove all concepts with stability below a fixed threshold. We computed the stability indices for concepts of our example from Fig. 1. There are 17 con-

Of course, stable concepts (i.e., satisfying the chosen stability threshold) do not always form a lattice. This may or may not be a problem. If all we need is a directly observable taxonomy of scientific fields, there seem to be no reason to request this taxonomy to be a lattice. Sometimes however we may like to have a lattice in order to be able to apply lattice-based analysis techniques. This issue is beyond the scope of the present paper; nonetheless, we suggest some possible strategies in Sect. 4.2.

Figure 2 presents a more readable epistemic taxonomy cepts with stability index above 0.5. Accidentally, they are closed under intersection of intents. Hence, they form a lattice, which is shown in Fig. 2.

representation, displaying the major fields of the community along with some meaningful joint communities (such as "mouse" and "human", as well as "signal, receptor"). However, some less important communities, like "mouse, conservation", "mammalian" or "signal, pathway, mouse", are also shown, and it cannot be seen from the picture that they are less important. Raising the stability threshold would eliminate these communities, but we would rather prefer having a multi-level representation with these communities rendered at a deeper level. 
Something similar applies, e.g., to the community "signal, receptor, growth, pathway", which is missing from Fig. 2, but is interesting according to the expert-based description of the field (see Sect. 2.2). In this respect, nested line diagrams should provide a handy representation by distinguishing various levels of importance of notions.

\subsection{Nested Line Diagrams}

Nested line diagrams are a well-established tool in formal concept analysis that makes it possible to distribute representation details across several levels [10]. The main idea is to divide the attribute set of the context into two (or more) parts, construct the concept lattices for generated subcontexts, and draw the diagram of one lattice inside each node of the other lattice. In the case of two parts, an inner concept $(A, B)$ enclosed within an outer concept $(C, D)$ corresponds to a pair $(A \cap C, B \cup D)$. Not every such pair is a concept of the original context. Only inner nodes that correspond to concepts are represented by circles; such nodes are said to be "realized". The outer diagram structures the data along one attribute subset, while the diagram inside an outer concept describes its structure in terms of the remaining attributes. For more details, see [10].

Partitioning the Attribute Set. The first step in constructing a nested line diagram is to split the attribute set into several parts. These parts do not have to be disjoint, but they will be in our case; hence, we are looking for a partition of the attribute set. As we seek to improve readability, we should display foremost the most significant attributes; therefore, we should assign major notions to higher levels, while leaving minor distinctions for lower levels. To this end, the words should be partitioned according to a "preference function", which could range from the simplest (e.g., word frequency within the corpus) to more complicated designs.

One could consider a minimal set of notions covering all authors, i.e., find an irredundant cover set, as words from such a set could be expected to play a key role in describing the community. In practice, we use the algorithm from [24]. We apply it iteratively: the first subcontext contains notions forming an irredundant cover set for the whole author set; the second subcontext includes notions not occurring in the first subcontext, while covering the set of authors excluding those that use only notions from the first subcontext, etc. The last level contains the remaining notions. Denoting by $\mathcal{I C}(\mathbb{K})$ an irredundant cover set of a context $\mathbb{K}$, we start with the context $\mathbb{K}_{0}=$ $\left(G_{0}, M_{0}, I_{0}\right)$ and, for $k>0$, recursively define the context $\mathbb{K}_{k}=\left(G_{k}, M_{k}, I_{k}\right)$, where $M_{k}=M_{k-1} \backslash \mathcal{I C}\left(\mathbb{K}_{k-1}\right)$, $G_{k}=\bigcup_{m \in M_{k}}\{m\}^{\prime}$, and $I_{k}=I \cap\left(G_{k} \times M_{k}\right)$. The sequence $\left(\mathcal{I C}\left(\mathbb{K}_{0}\right), \mathcal{I C}\left(\mathbb{K}_{1}\right), \ldots, \mathcal{I C}\left(\mathbb{K}_{n}\right), M_{0} \backslash M_{n}\right)$ for some $n>0$ defines a partition of the attribute set $M_{0}$ to be used for nesting.
In our example, "receptor", "growth", "signal", "brain", "mouse", and "human" cover the whole set of authors and constitute the outer-level subcontext notions. As we use only two levels, the inner-level subcontext is made of the remaining terms.

The resulting diagrams are shown in Fig. 3. Yet, while nesting makes it possible to distinguish between various levels of precision, both the outer and inner diagrams are still too large and recall the jumbled picture of Fig. 1. Stability-based pruning will address this problem; combining both procedures should yield a concise hierarchical representation.

\subsection{Combining Nesting and Stability- Based Pruning}

After partitioning the set of words and building lattices for individual parts, we prune each lattice using the stability criterion. We can use different thresholds for different parts depending on the number of concepts we are comfortable to work with. For our example, we get the two diagrams shown in Fig. 4. Many attributes of the inner diagram are not shown in the picture, as they are not contained in any stable intent.

We proceed by drawing one diagram inside the other and interpret the picture as usual. Again, only inner nodes corresponding to concepts of the full context are represented by circles (and said to be "realized"). Figure 5 shows the resulting structure for our context.

This approach may also help in reducing the computational complexity. Generally, computing inner concepts is the same as computing the lattice for the whole context, but, combining nesting and pruning, we compute inner nodes only for relevant (that is, non-pruned) outer nodes.

Let us denote by $\underline{\mathfrak{B}}_{p}(\mathbb{K})$ the set of concepts of $\mathbb{K}$ satisfying the chosen pruning criteria and ordered in the usual way (one may regard $p$ as an indicator of a specific pruning strategy). Assume that contexts $\mathbb{K}_{1}=\left(G, M_{1}, I_{1}\right)$ and $\mathbb{K}_{2}=\left(G, M_{2}, I_{2}\right)$ are subcontexts of $\mathbb{K}=(G, M, I)$ such that $M=M_{1} \cup M_{2}$ and $I=I_{1} \cup I_{2}$. We define the set of concepts corresponding to nodes of the nested line diagram of the pruned concept sets $\underline{\mathfrak{B}}_{p}\left(\mathbb{K}_{1}\right)$ and $\underline{\mathfrak{B}}_{p}\left(\mathbb{K}_{2}\right)$ :

$$
\begin{array}{r}
\underline{\mathfrak{B}}_{p}\left(G, M_{1}, M_{2}, I\right)=\{(A, B) \in \mathfrak{B}(\mathbb{K}) \mid \forall i \in\{1,2\}, \\
\left.\left(\left(B \cap M_{i}\right)^{\prime}, B \cap M_{i}\right) \in \underline{\mathfrak{B}}_{p}\left(\mathbb{K}_{i}\right)\right\}
\end{array}
$$

Proposition 2. If $\underline{\mathfrak{B}}_{p}\left(\mathbb{K}_{1}\right)$ and $\underline{\mathfrak{B}}_{p}\left(\mathbb{K}_{2}\right)$ are $\bigvee$ subsemilattices of $\underline{\mathfrak{B}}\left(\mathbb{K}_{1}\right)$ and $\underline{\mathfrak{B}}\left(\mathbb{K}_{2}\right)$, respectively, then $\underline{\mathfrak{B}}_{p}\left(G, M_{1}, M_{2}, I\right)$ is a $\bigvee$-subsemilattice of $\underline{\mathfrak{B}}(\mathbb{K})$ and the map

$$
(A, B) \mapsto\left(\left(\left(B \cap M_{1}\right)^{\prime}, B \cap M_{1}\right),\left(\left(B \cap M_{2}\right)^{\prime}, B \cap M_{2}\right)\right)
$$

is a $\bigvee$-preserving order embedding of $\mathfrak{B}_{p}\left(G, M_{1}, M_{2}, I\right)$ in the direct product of $\underline{\mathfrak{B}}_{p}\left(\mathbb{K}_{1}\right)$ and $\underline{\mathfrak{B}}_{p}\left(\mathbb{K}_{2}\right)$.

We omit the proof due to space restrictions. 

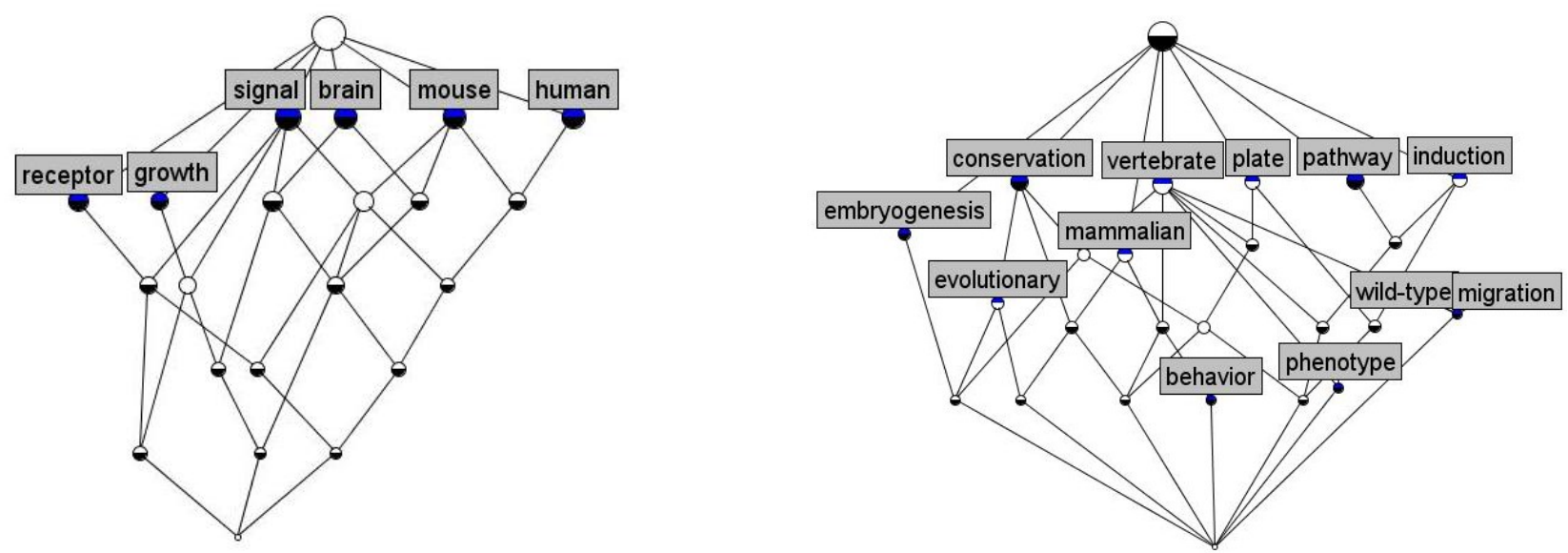

Figure 3: Outer and inner diagrams for the nested line diagram of the zebrafish context
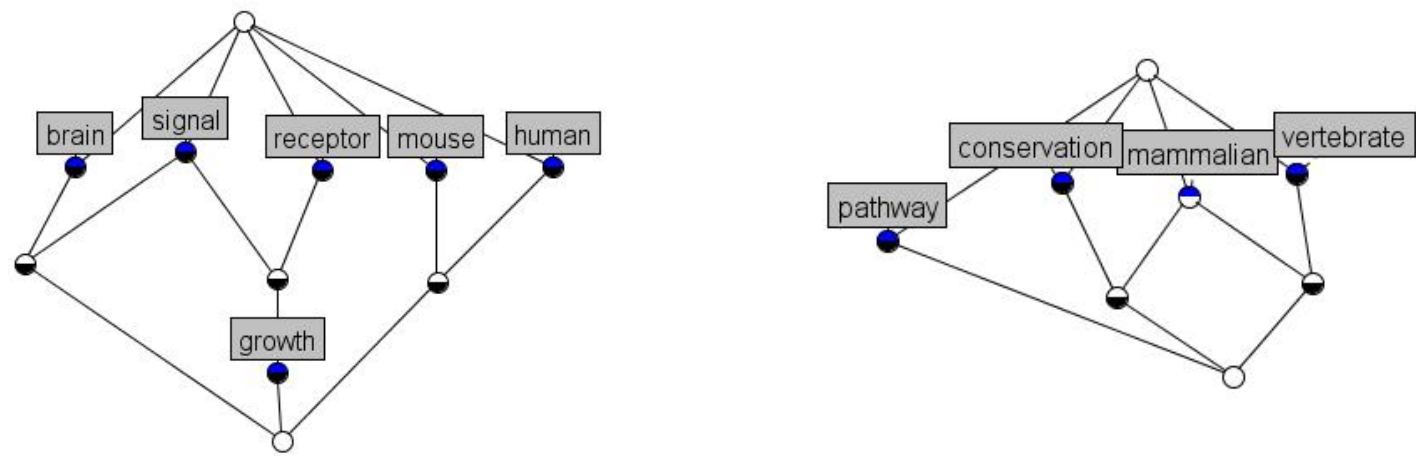

Figure 4: The pruned outer and inner lattices from Fig. 3 (resp. thresholds 0.70 and 0.54 )

Unlike in standard nesting [10], the component maps $(A, B) \mapsto\left(\left(B \cap M_{i}\right)^{\prime}, B \cap M_{i}\right)$ are not necessarily surjective on $\mathfrak{B}\left(\mathbb{K}_{i}\right)$. Hence, some outer nodes in our nested line diagram may be empty, i.e., contain no realized inner nodes, and some nodes of the inner diagram may never be realized.

Back to our example, the pruned outer diagram embraces most of the expert-based description outlined in Sect. 2.2 within a readable structure: it shows a joint focus on "human" and "mouse" (comparative studies); features several subfields made of "signal", "receptor", and "growth"; and displays brain studies, also in connection with signaling issues. The nested line diagram allows a deeper insight into the substructure of particular fields embedded within the pruned outer diagram. One may notice that outer nodes involving "human" and "mouse" show "vertebrate", "mammalian", and "conservation" in their inner diagrams, while outer nodes involving "signal" and "receptor" display "pathway", which is consistent with the real state of affairs.

\section{Further Work}

\subsection{Variants of Stability}

The stability index $\sigma$ as in Definition 1 and [9] refers to the stability of an intent; we may call it intensional. The extensional stability index of a concept $(A, B)$ can be defined similarly:

$$
\sigma_{e}(A, B)=\frac{\left|\left\{D \subseteq B \mid D^{\prime}=A\right\}\right|}{2^{|B|}} .
$$

The extensional stability of a concept is the probability of preserving its extent after leaving out an arbitrary number of attributes, and a proposition similar to Proposition 1 holds. Extensional stability relates to the social aspect of the concept, measuring how much the community as a group of people depends on a particular topic. It also allows one to fight noisy words - a community based on a noisy word (or, e.g., a homograph used differently within different communities) will be extensionally unstable.

Proposition 1 suggests how the general stability index of a concept $(A, B)$ should be defined - as the ratio between the number of subcontexts of $\mathbb{K}=(G, M, I)$ preserving the concept up to the omitted objects and at- 


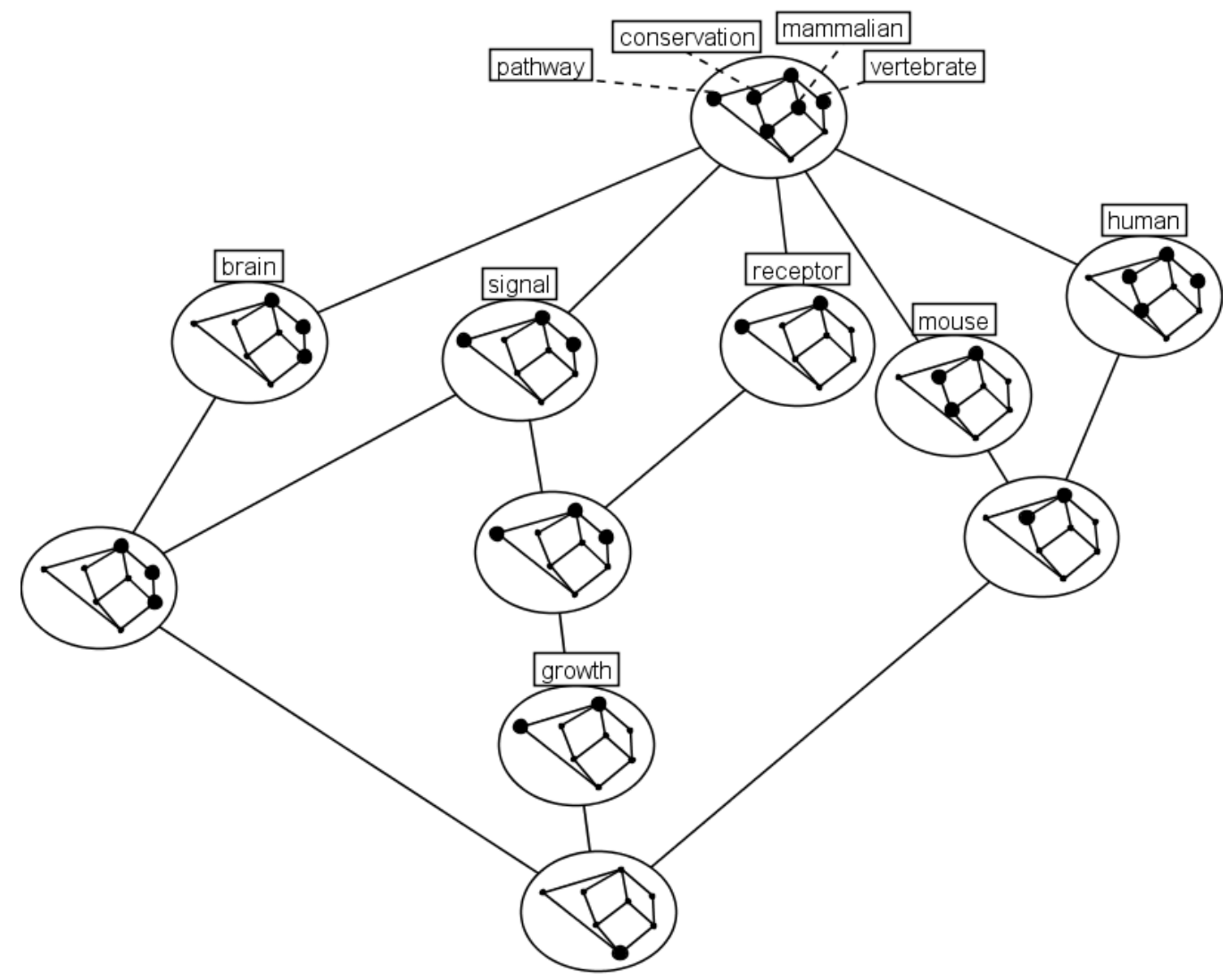

Figure 5: Nested line diagram of pruned lattices from Fig. 4

tributes and the total number of subcontexts, or, more formally:

$$
\frac{\left|\left\{(H, N, J) \mid \begin{array}{l}
H \subseteq G, N \subseteq M, J=I \cap(H \times N), \\
A_{H}^{J}=B_{N}, B_{N}^{J}=A_{H}
\end{array}\right\}\right|}{2^{|G|+|M|}}
$$

where $A_{H}=A \cap H$ and $B_{N}=B \cap N$. As of now, we are not aware of any realistic method for computing the general stability; thus, it is only of theoretical interest.

On the other hand, limited versions of stability (e.g., computed over subsets of a certain size only), as well as various combinations of extensional and intensional stability, are worth trying.

\subsection{Strategies for Pruning}

Other techniques aiming at reducing the number of concepts should be tested and, perhaps, some of them can be combined with stability for better results - notably pruning based on monotonous criteria like extent/intent size. Another method is given by attribute-dependency formulas [25], involving an expert-specified hierarchy on the attribute set (e.g., "human" and "mouse" are subtypes of "vertebrate").

As noticed in Sect. 3.2, pruning may not necessarily yield a lattice. We can handle this situation in several ways: for example, enlarging the resulting structure by including all intersections of stable intents or reducing it by eliminating some stable intents. Regarding the latter option, we may prefer to merge an unstable concept $(A, B)$ with its subconcept $(C, D)$ rather than simply drop $(A, B)$. It is not immediately clear how to choose $(C, D)-$ only that it should be somehow close to $(A, B)$. Merging can be done by assuming that all objects from $A$ have all attributes from $D$ and replacing the context relation $I$ by $I \cup A \times D$ (cf. [26]). However, the modified context may have intents that are absent from the initial context, which is probably undesirable. Alternatively, one could add $B \rightarrow D$ to the implication system of the context. The lattice of attribute subsets generated by this augmented implication system will be different from the original lattice only in that $B$ and possibly some of its previously closed supersets are not in the new lattice.

A different approach would involve merging based on partial implications (or association rules): compute all partial implications for the given confidence threshold and add them to the implication system of the context. It is a matter of further experiments to see which strategies are suitable for our goals. 


\subsection{Nesting}

Nested line diagrams are not limited to two levels, although it still has to be investigated whether multi-level diagrams remain readable and interpretable. Various techniques for partitioning attribute sets should be explored. One strategy specific to our application is to partition words according to their type: as a verb, noun, adjective; or as a method, object, property, etc. Such a method can be combined with other feature selection algorithms.

It should be noted that nesting seems to have more potential if used in interactive software tools that allow the user to zoom in and out on particular communities instead of having to deal with the entire picture. The fact that one need not compute everything at once provides an additional computational advantage.

\subsection{Dynamic Monitoring}

Modeling changes of the community structure should be particularly useful to describe historically the evolution of fields, either longitudinally or dynamically. The longitudinal approach means establishing a relation between community structures corresponding to different time points, e.g., identifying cases when several communities have merged into one or a community has divided into several sub-communities. FCA offers some methods for comparing two lattices built from identical objects and/or attributes (e.g., see [27]). Yet the relevance of such methods is likely to be application-dependent, and they should certainly be adapted for reduced lattice-based structures we work with.

A more ambitious dynamic approach to modeling changes assumes that any elementary change in the database (any modification of $G, M$, or $I$ ) should correspond to a concrete change in the representation of communities, and it should always be possible to trace a change in the community structure to a sequence of elementary changes in the database.

\section{Conclusion}

The approach discussed in this paper is based on the assumption that community structure in knowledge-based social networks should be dealt with more deeply than by simply relying on single-mode characterizations, as is often the case. In previous work [7,8], it was shown how concept lattices can be used to build knowledge taxonomies from data describing authors by sets of terms they use in their papers. As it frequently happens with concept lattices derived from real data, such taxonomies tend to be huge and, therefore, hard to compute and analyze. The computational complexity can be partially addressed by reducing the number of agents, since a taxonomy centered on knowledge fields rather than individ- uals justifies the use of a random representative sample of authors.

However, the interpretability of results requires a more serious effort. In this paper, we proposed a pruning method based on the stability indices of formal concepts [9]. We think that this method not simply reduces the concept lattice to a somewhat rougher structure, but also helps to fight noise in data, so that the resulting structure might even be more accurate in describing the knowledge community than the original lattice is.

We suggested that this method could also be applied to constituent parts of a nested line diagram in order to achieve an optimal relationship between the readability of the taxonomy and the level of detail in it. This is beneficial from the viewpoint of computational complexity, too: it is easier to compute the lattices of subcontexts used in nesting and then prune each of them individually than to compute the lattice of the entire context and prune it. Besides, nested line diagrams admit "lazy" computation: within an interactive software tool the user can choose which outer nodes to explore, then inner diagrams corresponding to neglected outer nodes will never be computed.

We have illustrated the proposed techniques with a small example. Of course, wider experiments are needed to see how this all works. There are quite a few open questions: how to efficiently compute stability, how exactly stability-based criteria should be formulated and applied (e.g., dropping unstable nodes vs. merging them with stable nodes), what other compression techniques exist and how they perform against stability-based pruning, etc. We have summarized some of the possible research directions in Sect. 4. Thus, this paper is only a first step towards a consistent methodology for creating concise knowledge taxonomies based on concept lattices.

\section{References}

[1] Schmitt, F., ed.: Socializing Epistemology: The Social Dimensions of Knowledge. Lanham, MD: Rowman \& Littlefield (1995)

[2] Leydesdorff, L.: In search of epistemic networks. Social Studies of Science 21 (1991) 75-110

[3] Haas, P.: Introduction: epistemic communities and international policy coordination. International Organization 46(1) (1992) 1-35

[4] McCain, K.W.: Cocited author mapping as a valid representation of intellectual structure. Journal of the American Society for Information Science 37(3) (1986) 111-122

[5] Atran, S.: Folk biology and the anthropology of science: Cognitive universals and cognitive particulars. Behavioral and Brain Sciences 21 (1998) 547-609 
[6] Lorrain, F., White, H.C.: Structural equivalence of individuals in social networks. Journal of Mathematical Sociology 1(49-80) (1971)

[7] Roth, C., Bourgine, P.: Epistemic communities: Description and hierarchic categorization. Mathematical Population Studies 12(2) (2005) 107-130

[8] Roth, C., Bourgine, P.: Lattice-based dynamic and overlapping taxonomies: the case of epistemic communities. Scientometrics 69(2) (2006)

[9] Kuznetsov, S.O.: On stability of a formal concept. In SanJuan, E., ed.: JIM, Metz, France (2003)

[10] Ganter, B., Wille, R.: Formal Concept Analysis: Mathematical Foundations. Springer, Berlin (1999)

[11] Girvan, M., Newman, M.E..J.: Community structure in social and biological networks. PNAS 99 (2002) $7821-7826$

[12] Noyons, E.C.M., van Raan, A.F.J.: Monitoring scientific developments from a dynamic perspective: self-organized structuring to map neural network research. Journal of the American Society for Information Science 49(1) (1998) 68-81

[13] White, D.R., Duquenne, V.: Social network \& discrete structure analysis: Introduction to a special issue. Social Networks 18 (1996) 169-172

[14] Freeman, L.: Cliques, Galois lattices, and the structure of human social groups. Social Networks 18 (1996) 173-187

[15] Falzon, L.: Determining groups from the clique structure in large social networks. Social Networks 22 (2000) 159-172

[16] Peyriéras, N. Personal communication. (2005)

[17] Grunwald, D.J., Eisen, J.S.: Headwaters of the zebrafish - emergence of a new model vertebrate. Nature Rev. Genetics 3(9) (2002) 717-724

[18] Bradbury, J.: Small fish, big science. PLoS Biology 2(5) (2004) 568-572
[19] Stumme, G., Taouil, R., Bastide, Y., Pasquier, N., Lakhal, L.: Computing iceberg concept lattices with TITANIC. Data and Knowledge Engineering 42 (2002) 189-222

[20] Kuznetsov, S.O., Obiedkov, S.: Comparing performance of algorithms for generating concept lattices. J. Expt. Theor. Artif. Intell. 14(2/3) (2002) 189-216

[21] Bayardo, Jr., R., Goethals, B., Zaki, M., eds.: Proc. of the IEEE ICDM Workshop on Frequent Itemset Mining Implementations (FIMI 2004), CEURWS.org (2004)

[22] Kuznetsov, S.O.: Stability as an estimate of the degree of substantiation of hypotheses derived on the basis of operational similarity. Nauchn. Tekh. Inf., Ser.2 (Automat. Document. Math. Linguist.) (12) (1990) 21-29

[23] Kohavi, R.: A study of cross-validation and bootstrap for accuracy estimation and model selection. In: IJCAI. (1995) 1137-1145

[24] Batni, R.P., Russell, J.D., Kime, C.R.: An efficient algorithm for finding an irredundant set cover. Journal of the Association for Computing Machinery 21(3) (1974) 351-355

[25] Belohlávek, R., Sklenar, V.: Formal concept analysis constrained by attribute-dependency formulas. In Ganter, B., Godin, R., eds.: ICFCA 2005. Volume 3403 of LNAI. (2005) 176-191

[26] Rome, J.E., Haralick, R.M.: Towards a formal concept analysis approach to exploring communities on the world wide web. In Ganter, B., Godin, R., eds.: ICFCA 2005. Volume 3403 of LNAI. (2005) 33-48

[27] Wille, R.: Conceptual structures of multicontexts. In Eklund, P., Ellis, G., Mann, G., eds.: Conceptual Structures: Knowledge Representation as Interlingua. Volume 1115 of LNAI., Heidelberg-Berlin-New York, Springer (1996) 23-29 